\title{
SAKURAI'S OBJECT: CONSTRAINTS FROM ITS VARIABILITY?
}

\author{
A. GAUTSCHY \\ Astronomisches Institut der Univ. Basel, Switzerland \\ gautschy@astro.unibas.ch \\ H.W. DUERBECK \\ Space Telescope Science Institute, Baltimore, USA \\ duerbeck@stsci.edu \\ A.M. VAN GENDEREN \\ Leiden Observatory, Leiden, Netherlands \\ genderen@strw.LeidenUniv.nl \\ AND \\ S. BENETTI \\ European Southern Observatory, Santiago, Chile \\ benetti@eso.org
}

The peculiar outburst of the star baptized Sakurai's Object (SO) is a conceivable example of a late He shell flash in a post-AGB object. The new source of nuclear energy forces such objects toward high luminosities and eventually low effective temperatures; they cross the HR diagram in a comparable fashion as FG Sge did in the past - i.e., they move noticeably on the HR diagram on human timescales. From monitoring campaigns of SO during the last year, first estimates of its cooling rate were derived and in particular cyclic light variability was established. We present first results from attempts to model stellar envelopes appropriate for SO. As we hypothesize the light variability to be attributable to stellar pulsations, we aim at constraining the basic stellar parameters based on stability analyses of our envelope models. Radial, nonadiabatic stability comrputations provided predictions of the modal content which should be observable as SO evolves. The particular components in such mode spectra of SO as they are to appear in the coming years should indeed help to constrain basic stellar parameters such as mass and luminosity. 